\title{
MCDHF calculations of isotope shifts in neutral antimony
}

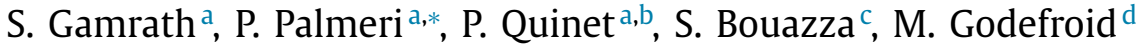 \\ a Physique Atomique et Astrophysique, Université de Mons - UMONS, Mons B-7000, Belgium \\ ${ }^{\mathrm{b}}$ IPNAS, Université de Liège, Liège B-4000, Belgium \\ c Département de Physique, Université de Reims-Champagne-Ardenne, UFR SEN, BP 1039, Reims Cedex 2 F-51687, France \\ ${ }^{\mathrm{d}}$ Chimie Quantique et Photophysique, Université libre de Bruxelles, Brussels B-1050, Belgium
}

\section{A R T I C L E I N F O}

\section{Article history:}

Received 8 May 2018

Revised 5 July 2018

Accepted 5 July 2018

Available online 6 July 2018

\section{Keywords:}

Isotope shift

Relativistic effects

Neutral antimony

Fine structure

\begin{abstract}
A B S T R A C T
$A b$ initio multiconfiguration Dirac-Hartree-Fock (MCDHF) calculations have been carried out in order to determine the isotope shift (IS) electronic parameters of transitions belonging to electric dipole (E1) transition arrays $5 s^{2} 5 p^{3}-5 s^{2} 5 p^{2} 6 s, 5 s^{2} 5 p^{2} 6 s-5 s^{2} 5 p^{2} 6 p$ and $5 s^{2} 5 p^{2} 6 s-5 s^{2} 5 p^{2} 7 p$ in neutral antimony, Sb I. In a correlation model limited to single and double excitations from the valence shells, these parameters, combined with the changes in mean-square nuclear charge radius $\delta\left\langle r^{2}\right\rangle^{123,121}$ compiled by Angeli and Marinova [3] produce isotope shifts values in good agreement with the most recent measurements by high-resolution emission and optogalvanic absorption spectroscopy of Sobolewski et al. [5] but not with the old measurements of Buchholz et al. [4] for $5 p^{3}-5 p^{2} 6 s$. However, our analysis does not allow to reject the latter due to the large uncertainty affecting $\delta\left\langle r^{2}\right\rangle^{123,121}$, i.e. $0.072 \pm 0.048 \mathrm{fm}^{2}$ [3]. This shows the need of a more accurate determination of this nuclear parameter. Although improving excitation energies, the inclusion of core-valence correlation limited to one hole in the $4 \mathrm{~d}$ core subshell destroyed the theory-experiment agreement on the IS parameters.
\end{abstract}

(c) 2018 Elsevier Ltd. All rights reserved.

\section{Introduction}

High-resolution spectroscopic measurements of isotope shifts (IS) of atomic spectral lines are carried out to test the hyperfine atomic structures, to deduce the changes in the nuclear meansquare charge radius, to give information on electron densities inside the nuclear volume and to estimate the balance between mass and volume contributions. These measurements can have a strong impact in nuclear physics. As a recent example, the optical laser technique has been used at ISOLDE (CERN) in order to measure the charge radii of radioactive isotopes $49,51,52 \mathrm{Ca}$ resulting in unexpectedly large values for these neutron-rich calcium isotopes beyond the magic number 28 and challenging the doubly magic nature of ${ }^{52} \mathrm{Ca}$ [1].

Antimony (Sb), the element with $Z=51$ of the Periodic Table, has only two stable isotopes, ${ }^{121} \mathrm{Sb}$ and ${ }^{123} \mathrm{Sb}$, with natural abundances of $57.21 \%$ and $42.79 \%$ respectively. Besides these natural species, 36 radioactive isotopes are known with mass numbers $A$ ranging from 103 to 140 and with corresponding half-lives spanning from $93 \mathrm{~ms}\left({ }^{139} \mathrm{Sb}\right)$ to 2.7586 years $\left({ }^{125} \mathrm{Sb}\right)$ [2]. By contrast, the mean nuclear charge radius, $\left\langle r^{2}\right\rangle^{1 / 2}$, is known only for

\footnotetext{
* Corresponding author.

E-mail address: patrick.palmeri@umons.ac.be (P. Palmeri).
}

the two stable isotopes, i.e. $4.6802 \pm 0.0026 \mathrm{fm}$ for ${ }^{121} \mathrm{Sb}$ and $4.6879 \pm 0.0025 \mathrm{fm}$ for ${ }^{123} \mathrm{Sb}$ [3]. The balance between the mass shift and field shift contributions depends on the nature of the electronic transition and on the considered isotope pair. For an intermediate $Z$ element such as Sb I, both contributions can be relevant, rending precise calculations of IS challenging and their comparison with experiment interesting for extracting nuclear quantities.

Moreover, as far as the authors know, there are only two measurements of IS available in the literature $[4,5]$. They both concern exclusively the stable ${ }^{121},{ }^{123} \mathrm{Sb}$ isotopes. The older one dates from the seventies by Buchholz et al. [4] who determined the IS of nine E1 transitions of ${ }^{121,123} \mathrm{Sb}$ I connecting the configurations $5 s^{2} 5 p^{3}$ and $5 s^{2} 5 p^{2} 6 s$ and estimated for the first time the change in mean-square nuclear charge radius between these two isotopes, $\delta\left\langle r^{2}\right\rangle^{123,121} \equiv\left(\left\langle r^{2}\right\rangle_{123}^{1 / 2}\right)^{2}-\left(\left\langle r^{2}\right\rangle_{121}^{1 / 2}\right)^{2}=0.12 \pm 0.04 \mathrm{fm}^{2}$. The latter is almost a factor of two larger than the value deduced from the mean nuclear charge radii of both isotopes given in Angeli and Marinova's compilation [3], i.e. $0.072 \pm 0.048 \mathrm{fm}^{2}$.

More recently, Sobolewski et al. [5] extended the available experimental IS data in neutral antimony by measuring eight lines belonging to the E1 transition arrays $5 s^{2} 5 p^{3}-5 s^{2} 5 p^{2} 6 s$, $5 s^{2} 5 p^{2} 6 s-5 s^{2} 5 p^{2} 6 p$ and $5 s^{2} 5 p^{2} 6 s-5 s^{2} 5 p^{2} 7 p$. Large discrepancies have been found between the two sets of experimental IS for the three common lines at $\lambda=363.8,372.3$ and $403.4 \mathrm{~nm}$. Their IS 
measurements disagree with Buchholz et al. [4] for one common line $5 s^{2} 5 p^{32} \mathrm{P}_{3 / 2}^{0}-5 s^{2} 5 \mathrm{p}^{2} 6 \mathrm{~s}^{4} \mathrm{P}_{3 / 2}$ (NIST term designations [6] are used from here onward) at $363.8 \mathrm{~nm}$ by a factor $\simeq 2$ but were supported by their theoretical investigations. The latter were based on the use of the scaling law to estimate the transition normal mass shift (NMS) from the wavelength of the optical transition, and on theoretical estimations of the specific mass shift (SMS) and field shift (FS) contributions. The agreement between their experimental IS and their calculated IS was found to be rather satisfactory, except for the transition $5 s^{2} 5 p^{2} 6 s^{4} P_{3 / 2}-5 s^{2} 5 p^{2} 6 p(1,3 / 2)_{5 / 2}^{0}$ at $1074.2 \mathrm{~nm}$ where a discrepancy factor $\sim 2$ was observed. In Sobolewski et al. [5], two different approaches were used. On one hand, the Vinti integrals involved in the SMS contribution were computed using the pseudo-relativistic Hartree-Fock (HFR) method of Cowan [7] without distinguishing radially the $p_{1 / 2}$ and $p_{3 / 2}$ electrons. On the other, the electron densities at the nucleus were obtained using the pseudo-relativistic Pennsylvania State University Hartree-Fock (PSUHFR) code [8] for the FS. In their approach, the change of the mean-square nuclear charge radius $\delta\left\langle r^{2}\right\rangle^{123,121}$ was not used explicitly thanks to an indirect normalisation procedure. The FS contributions were indeed determined for the different lines considered by rescaling charge electron density differences using the $372.3 \mathrm{~nm}$ transition as the reference line. For the latter, the FS factor was extracted from the observed residual IS combined with theoretical estimations of the SMS contribution.

In the present paper, following previous IS studies [9,10], we have used the fully relativistic multiconfiguration Dirac-HartreeFock (MCDHF) method [11] to calculate the IS electronic parameters, $K_{R N M S}, K_{R S M S}$ and $F$, for 49 fine-structure levels belonging to the configurations $5 s^{2} 5 p^{3}, 5 s^{2} 5 p^{2} 6 s, 5 s^{2} 5 p^{2} 6 p$ and $5 s^{2} 5 p^{2} 7 p$ in $\mathrm{Sb}$ I. These parameters can be used to determine IS of the transitions involving these levels for any pair of $\mathrm{Sb}$ isotopes along with their different NMS, SMS and FS contributions or the corresponding changes in mean-square nuclear charge radius $\delta\left\langle r^{2}\right\rangle^{A, A^{\prime}}$. In Section 2, the computational model is detailed. Our results are presented and discussed in Section 3 and the conclusions are given in the last section.

\section{Theoretical method and calculations}

The MCDHF calculations have been carried out using the GRASP2K atomic structure package version 1_1 [11] along with its isotope shift module RIS3 [12]. A description of the method is given below.

\subsection{Isotope shifts}

The frequency spectral transition $v_{u l}$ connecting an upper level $E_{u}$ to a lower level $E_{l}$ is given by

$v_{u l}=\frac{E_{u}-E_{l}}{h}$

It differs from one isotope of mass $M$ to another of mass $M^{\prime}$ by

$\delta v_{u l}^{M, M^{\prime}} \equiv v_{u l}^{M}-v_{u l}^{M^{\prime}}=\frac{\delta E_{u}^{M, M^{\prime}}-\delta E_{l}^{M, M^{\prime}}}{h}$

with

$\delta E_{i}^{M, M^{\prime}} \equiv E_{i}^{M}-E_{i}^{M^{\prime}} \quad(i=u, l)$

and can be parameterized (with $M>M^{\prime}$ ) [12] as

$\delta v_{u l}^{M, M^{\prime}}=\left(\frac{M^{\prime}-M}{M M^{\prime}}\right) \Delta \tilde{K}_{u l}+F_{u l} \delta\left\langle r^{2}\right\rangle^{M, M^{\prime}}$

where $\Delta \tilde{K}_{u l}=\tilde{K}_{u}-\tilde{K}_{l}$ refers to the mass-shift electronic parameter, $F_{u l}=F_{u}-F_{l}$ is the field-shift electronic factor and $\delta\left\langle r^{2}\right\rangle^{M, M^{\prime}} \equiv$ $\left\langle r^{2}\right\rangle^{M}-\left\langle r^{2}\right\rangle^{M^{\prime}}$ is the mean-square nuclear charge radius difference between the two isotopes.

The relativistic mass-shift electronic parameter $\tilde{K}_{R M S}$ for a level is related to the expectation value of the relativistic recoil Hamiltonian, $H_{R M S}$,

$\tilde{K}_{R M S}=\frac{M}{h}\left\langle\Psi\left|H_{R M S}\right| \Psi\right\rangle$

or, in energy units,

$K_{R M S}=h \tilde{K}_{R M S}$

where $H_{R M S}$ can be split into a one-body, the relativistic normal mass-shift (RNMS), operator and a two-body, the relativistic specific mass-shift (RSMS), operator [13-15]

$H_{R M S}=H_{R N M S}+H_{R S M S}$

$H_{R N M S}=\frac{1}{2 M} \sum_{i}\left[p_{i}^{2}-\frac{\alpha Z}{r_{i}}\left[\vec{\alpha}_{i}+\frac{\left(\vec{\alpha}_{i} \cdot \vec{r}_{i}\right) \vec{r}_{i}}{r_{i}^{2}}\right] \cdot \vec{p}_{i}\right]$

$H_{R S M S}=\frac{1}{2 M} \sum_{i \neq j}\left[\vec{p}_{i} \cdot \vec{p}_{j}-\frac{\alpha Z}{r_{i}}\left[\vec{\alpha}_{i}+\frac{\left(\vec{\alpha}_{i} \cdot \vec{r}_{i}\right) \vec{r}_{i}}{r_{i}^{2}}\right] \cdot \vec{p}_{j}\right]$

The field-shift (FS) electronic factor $F_{u l}$ is related to the change of the electronic total probability density, $\Delta|\Psi(\overrightarrow{0})|_{u l}^{2} \equiv \rho_{u}^{e}(\overrightarrow{0})-$ $\rho_{l}^{e}(\overrightarrow{0})$, at the origin between the two levels involved in the transition [12]

$F_{u l}=\frac{Z}{3 \hbar}\left(\frac{e^{2}}{4 \pi \epsilon_{0}}\right) \Delta|\Psi(\overrightarrow{0})|_{u l}^{2}$

The total probability electron density at the origin is defined for a level by

$\rho^{e}(\overrightarrow{0}) \equiv\left\langle\Psi\left|\sum_{i} \delta\left(\vec{r}_{i}\right)\right| \Psi\right\rangle$

\subsection{The MCDHF approach}

In the MCDHF method [11], the atomic state function (ASF), $\Psi$, appearing in Eqs. (5) and (11) is represented by a linear combination of configuration state functions (CSFs), $\Phi$, with the same parity, $\Pi$, total angular momentum and total magnetic quantum numbers, $J$ and $M_{J}$, as

$\Psi\left(\Pi J M_{J}\right)=\sum_{i} c_{i} \Phi\left(\gamma_{i} \Pi J M_{J}\right)$

where $\left\{c_{i}\right\}$ are the mixing coefficient, $\gamma_{i}$ stands for all other quantum numbers needed to unambiguously specify the CSF built from one-electron spin-orbitals, $\phi_{n \kappa m}$, of the form:

$\phi_{n \kappa m}(r, \theta, \varphi)=\frac{1}{r}\left(\begin{array}{c}P_{n \kappa}(r) \chi_{\kappa m}(\theta, \varphi) \\ i Q_{n \kappa}(r) \chi_{-\kappa m}(\theta, \varphi)\end{array}\right)$.

$P_{n \kappa}(r)$ and $Q_{n \kappa}(r)$ are, respectively, the large and the small radial components of the wave functions, and the angular functions $\chi_{\kappa m}(\theta, \varphi)$ are the spin or spherical harmonics [16]. The quantum number $\kappa$ is given by:

$\kappa= \pm\left(j+\frac{1}{2}\right)$,

where $\kappa=-(j+1 / 2) a$, with $a$ defined so that

$\ell=j-\frac{1}{2} a ; \quad a= \pm 1$.

The radial functions $P_{n \kappa}(r)$ and $Q_{n \kappa}(r)$ are numerically represented on a logarithmic grid and are required to be orthonormal within each $\kappa$ symmetry. In the MCDHF variational procedure, the radial 
functions and the expansion coefficients $c_{i}$ are optimized to selfconsistency [17]. A spherical model, here a Fermi nucleus, has been chosen for the nuclear charge distribution

$\rho(r)=\frac{\rho_{0}}{1+e^{(r-c) / d}}$,

where $\rho_{0}$ is a normalisation constant, $c$ the half-density of the nuclear charge distribution and $d=t /(4 \ln (3))$ is related to the nuclear surface thickness $t$, with $c$ computed according to Parpia and Mohanty [18] and $t=2.30 \mathrm{fm}$. The field shift electronic factors (10) have been estimated in the first-order perturbation approximation using the electron wave function calculated for the reference isotope $A=121$ using the prescriptions of Nazé et al. [12].

\subsection{Computational strategy}

The concept of the restricted active space (RAS) [19] method can be used for building MCDHF multiconfiguration expansions with a given orbital active set [17]. The CSF expansions defining the configuration space are produced by exciting the electrons from the reference configurations to a given set of orbitals. The rules adopted for generating the configuration space differ according to the correlation model used. Within a given correlation model, the active set of orbitals spanning the configuration space is increased in a systematic way to monitor the convergence of the total energies and the isotope shift electronic parameters.

Our calculations have been focused on the fine structure levels participating in the E1 transition arrays $5 s^{2} 5 p^{3}-5 s^{2} 5 p^{2} 6 s$, $5 s^{2} 5 p^{2} 6 s-5 s^{2} 5 p^{2} 6 p$ and $5 s^{2} 5 p^{2} 6 s-5 s^{2} 5 p^{2} 7 p$ in $S b$ I $(Z=51)$.The reference isotope has been chosen to be the most abundant stable isotope with mass number $A=121$ [20]. Two different models (A and $\mathrm{B}$ ) have been considered in the present work.

\subsubsection{Model A}

The calculations of the MCDHF wave functions have been carried out in seven steps for each parity. For the odd parity levels, these steps were the following:

- Step 0: the core orbitals, i.e. $1 \mathrm{~s}$ to $4 \mathrm{~d}$, along with the $5 \mathrm{~s}, 5 \mathrm{p}$, $6 p$ and $7 p$ orbitals, have been optimized. All the 43 CSFs belonging to the odd-parity interacting configurations $5 \mathrm{~s}^{2} 5 \mathrm{p}^{3}+$ $5 s^{2} 5 p^{2} 6 p+5 s^{2} 5 p^{2} 7 p$ with symmetries $J=1 / 2-5 / 2$ were retained in the configuration space. The energy functional was built within the framework of the average level (AL) option [16]. This option is most suitable for cases where all the eigenvalues of the hamiltonian are optimized at once. This is the case here as we want to obtain simultaneously all the 43 ASFs belonging to $5 s^{2} 5 p^{3}+5 s^{2} 5 p^{2} 6 p+5 s^{2} 5 p^{2} 7 p ~ J=1 / 2-5 / 2$.

- Step 1: the configuration space was increased to 17744 CSFs by considering all the single and double electron excitations to the $4 f, 5 s, 5 p, 5 d, 5 f, 5 g, 6 p$ and $7 p$ orbitals from the active orbitals $5 s, 5 p, 6 p$ and $7 p$ of the reference configurations $\left(5 s^{2} 5 p^{3}+5 s^{2} 5 p^{2} 6 p+5 s^{2} 5 p^{2} 7 p\right) J=1 / 2-5 / 2$. The $4 f, 5 d, 5 f$ and $5 \mathrm{~g}$ orbitals have been optimized, fixing all the others to the ones of Step 0 using an energy functional built from the lowest 43 ASFs within the framework of the extended optimal level (EOL) option [16].

- Steps 2-6: the configuration space has been extended to 58101 , 122 924, 228 665, 367122 and 538925 CSFs, respectively, using the same optimization procedure as described in Step 1, but adding single and double excitations to the $\{4 \mathrm{f}, n=5-6\}$, $\{4 \mathrm{f}, n=5-7 \mathrm{~h}\},\{4 \mathrm{f}, n=5-8 \mathrm{~h}\},\{4 \mathrm{f}, n=5-9 \mathrm{~h}\}$ and $\{4 \mathrm{f}, n=$ $5-10 \mathrm{~h}\}$ orbital sets successively ( $n \mathrm{~h}$ indicates that the maximum $\ell$-value considered is $\ell_{\max }=5$ ).

Concerning the even parity levels, the seven computational steps are detailed below:
- Step 0: similarly to the odd parity, the core orbitals, i.e. $1 \mathrm{~s}$ to $4 \mathrm{~d}$, along with the $5 \mathrm{~s}, 5 \mathrm{p}$ and $6 \mathrm{~s}$ orbitals, have been optimized. All the 6 CSFs belonging to the even-parity configuration $5 s^{2} 5 p^{2} 6 s$ with symmetries $J=1 / 2-3 / 2$ were retained in the configuration space. The energy functional was built within the framework of the average level (AL) option.

- Step 1: the configuration space was increased to 1942 CSFs by considering all the single and double electron excitations to the $4 \mathrm{f}, 5 \mathrm{~s}, 5 \mathrm{p}, 5 \mathrm{~d}, 5 \mathrm{f}, 5 \mathrm{~g}$ and $6 \mathrm{~s}$ orbitals from the active orbitals $5 s, 5 p$ and $6 s$ of the reference configurations $\left(5 s^{2} 5 p^{2} 6 s\right) J=$ $1 / 2-3 / 2$. The $4 \mathrm{f}, 5 \mathrm{~d}$, $5 \mathrm{f}$ and $5 \mathrm{~g}$ orbitals have been optimized, fixing all the others to the values of Step 0 using an energy functional built from the lowest 6 ASFs within the framework of the extended optimal level (EOL) option.

- Steps 2-6: the configuration space has been extended to 7732 , 18 103, 32 878, 52057 and 87152 CSFs, respectively, using the same optimization procedure as described in Step 1, but adding single and double excitations to the $\{4 \mathrm{f}, n=5-6\},\{4 \mathrm{f}, n=$ $5-7 \mathrm{~h}\},\{4 \mathrm{f}, n=5-8 \mathrm{~h}\},\{4 \mathrm{f}, n=5-9 \mathrm{~h}\}$ and $\{4 \mathrm{f}, n=5-10 \mathrm{~h}\}$ orbital sets successively ( $n \mathrm{~h}$ indicates that the maximum $\ell$ value considered is $\ell_{\max }=5$ ).

For the calculation of final IS between the two ${ }^{123,121} \mathrm{Sb}$ stable isotopes, the mean nuclear charge radii of both isotopes as given in the compilation of Angeli and Marinova [3] have been considered, corresponding to $\delta\left\langle r^{2}\right\rangle^{123,121}=0.072 \pm 0.048 \mathrm{fm}^{2}$.

\subsubsection{Model B}

In order to investigate the effects of extending the reference configurations and opening the core subshells, a relativistic configuration interaction (RCI) calculation has been carried out where the orbitals were fixed to those of step 4 of our Model A described in the previous section, i.e. with an orbital set limited up to $8 \mathrm{~h}$.

First, the reference space of Model A was extended by considering all single and double electron substitutions from the subshells $5 s$ and $5 p$ to the set of orbitals $\{4 f, 5 s, 5 p, 5 d, 5 f\}$. Those configurations that strongly interact with the spectroscopic configurations, i.e. $\left(5 s^{2} 5 p^{3}+5 s^{2} 5 p^{2} 6 p+5 s^{2} 5 p^{2} 7 p\right) J=1 / 2-5 / 2$ and $\left(5 s^{2} 5 p^{2} 6 s\right) J=1 / 2-3 / 2$, i.e., those with an absolute value of the mixing coefficient greater than 0.1 , were ultimately retained in the multi-reference.

More explicitly, they were $\left(5 p^{5}+5 p^{4} 6 p+5 p^{4} 7 p+\right.$ $\left.5 \mathrm{~s} 5 \mathrm{p}^{2} 5 \mathrm{~d} 6 \mathrm{p}+5 \mathrm{~s} 5 \mathrm{p}^{2} 5 \mathrm{~d} 7 \mathrm{p}+5 \mathrm{~s}^{2} 5 \mathrm{~d}^{2} 7 \mathrm{p}\right) J=1 / 2, \quad\left(5 \mathrm{p}^{5}+5 \mathrm{p}^{4} 6 \mathrm{p}+\right.$ $\left.5 \mathrm{p}^{4} 7 \mathrm{p}+5 \mathrm{~s} 5 \mathrm{p}^{2} 5 \mathrm{~d} 6 \mathrm{p}+5 \mathrm{~s} 5 \mathrm{p}^{2} 5 \mathrm{~d} 7 \mathrm{p}+5 \mathrm{~s}^{2} 5 \mathrm{~d}^{2} 6 \mathrm{p}+5 \mathrm{~s}^{2} 5 \mathrm{~d}^{2} 7 \mathrm{p}\right) J=3 / 2$, $\left(5 s 5 p^{2} 5 d 6 p+5 s 5 p^{2} 5 d 7 p\right) J=5 / 2$ in the odd parity, and $\left(5 s 5 p^{2} 5 d 6 s+5 s^{2} 5 d^{2} 6 s+5 p^{4} 6 s\right) J=1 / 2, \quad\left(5 s 5 p^{2} 5 d 6 s\right) J=3 / 2$ in the even parity. The total sizes of the multi-reference were 69 CSFs in the odd parity and 12 CSFs in the even parity.

Second, the configuration spaces were expanded from the above-mentioned multi-reference by including single and double excitations to unoccupied orbitals up to $8 \mathrm{~h}$ where the active set of orbitals was \{4d, 4f, 5s, 5p, 5d, 5f, 5g, 6s, 6p, 6d, 6f, 6g, 6h, 7s, $7 \mathrm{p}, 7 \mathrm{~d}, 7 \mathrm{f}, 7 \mathrm{~g}, 7 \mathrm{~h}, 8 \mathrm{~s}, 8 \mathrm{p}, 8 \mathrm{~d}, 8 \mathrm{f}, 8 \mathrm{~g}, 8 \mathrm{~h}$ \} and with the restriction of maximum one hole in the $4 \mathrm{~d}$ core orbital. The latter restriction ensures the inclusion of some core-valence correlation limited to the opening of the $4 \mathrm{~d}$ core subshell. These excitations generated 1946916 CSFs in the odd parity and 295,219 CSFs in the even parity. The configuration space was then reduced in order to render the RCI calculation feasible by keeping the CSFs that directly interact with at least one of the 81 multi-reference CSFs [24]. This led to 581,258 CSFs in the odd parity and 98,548 CSFs in the even parity.

Finally, the same mean radii as those used in Model A have been considered for the calculation of the final IS between the two ${ }^{123,121}$ Sb stable isotopes. 


\section{Results and discussion}

In Table 1, the electronic isotope shift parameters are presented for all the known fine-structure levels belonging to the odd $\left(5 p^{3}+5 p^{2} 6 p+5 p^{2} 7 p\right) J=1 / 2-5 / 2$ and even $5 p^{2} 6 s J=1 / 2-3 / 2$ configurations along with their corresponding calculated and experimental energies taken from Hassini et al. [25] as reported in the NIST database [6]. The average deviations of our ab initio MCDHF level energies with respect to the experimental values of Hassini et al. [25] are $1656 \mathrm{~cm}^{-1}$ and $407 \mathrm{~cm}^{-1}$ for Model A and Model B, respectively, showing an important improvement of the level energies when extending the reference configurations and considering some core-valence correlation by opening the ionic core.

Concerning the level isotope shift parameters shown in this table, one can observe the large dominance of the normal mass shift contribution over the specific mass shift by a factor of about 3, with opposite signs for these two mass contributions. Also, one notices differences between the results of Model A and Model B. These electronic parameters can be used to derive the isotope shifts of any transitions between these levels of any $\mathrm{Sb}$ I isotope pairs via Eq. (4).

In what follows, we will focus on the E1 transitions measured by Sobolewski et al. [5] and Buchholz et al. [4]. The MCDHF electronic parameters along with the corresponding isotope shifts (IS) between the ${ }^{123} \mathrm{Sb}$ and ${ }^{121} \mathrm{Sb}$ stable isotopes are given in Table 2 and are compared with the experimental values $[4,5]$ for these lines. One can see that our Model A IS values are supported by the measurements of Sobolewski et al. [5], within their uncertainties for most of the lines. Except for the transition $5 \mathrm{p}^{32} \mathrm{P}_{1 / 2}^{\mathrm{o}}-5 \mathrm{p}^{2}\left({ }^{3} \mathrm{P}\right) 6 \mathrm{~s}^{4} \mathrm{P}_{1 / 2}$ at $\lambda=372.3 \mathrm{~nm}$, striking differences (a factor of two or more) are seen between the experimental values of Buchholz et al. [4] and those of our Model A. Nonetheless, due to the large uncertainty affecting $\delta\left\langle r^{2}\right\rangle^{123,121}$, i.e. $\pm 0.048 \mathrm{fm}^{2}$ [3] which represents a relative error of more than $50 \%$, one cannot reject the former measurements. For instance, taking account this uncertainty, our Model A total IS would range from $125 \mathrm{MHz}$ to $401 \mathrm{MHz}$ for the transition $5 \mathrm{p}^{32} \mathrm{D}_{5 / 2}^{\mathrm{o}}-5 \mathrm{p}^{2}\left({ }^{3} \mathrm{P}\right) 6 \mathrm{~s}^{4} \mathrm{P}_{3 / 2}$ at $\lambda=277.0 \mathrm{~nm}$, to be compared with $96 \pm 60 \mathrm{MHz}$ of Buchholz et al. [4]. This illustrates the need of a more precise determination of the change in mean-square nuclear charge radius between these two isotopes.

By contrast, our Model B IS values are completely off of measurements by Sobolewski et al. [5] and of most of the experimental values by Buchholz et al. [4], in some occurrences by factors of one order of magnitude. This is the consequence of significant differences between the isotope shift parameters calculated with our two models with corresponding factors and order of magnitude variations, due to the incomplete inclusion of electron correlation contributions involving the core. The relatively small discords between our Model A IS and the experimental values of Sobolewski et al.most likely reveal the large cancellation between the limited core-valence correlation effects introduced through Model B and the remaining omitted correlation contributions.

At this point, one should bear in mind that the multireference space is quite limited and that the $[\mathrm{Kr}]$ core is kept closed to keep the size of the CSF expansions manageable in Model B. Unfortunately, both contributions were not dissociated in our analysis. The fact that the excitation energies have improved when going from Model A to Model B while the IS electronic parameters are deteriorated reveals that the considered correlation excitations affect differently the energy and the expectation values, with their differences, of the relevant mass and field shift operators. A similar situation has been met recently in Al I [26] where extended correlation (there the addition of core-core interactions) has improved

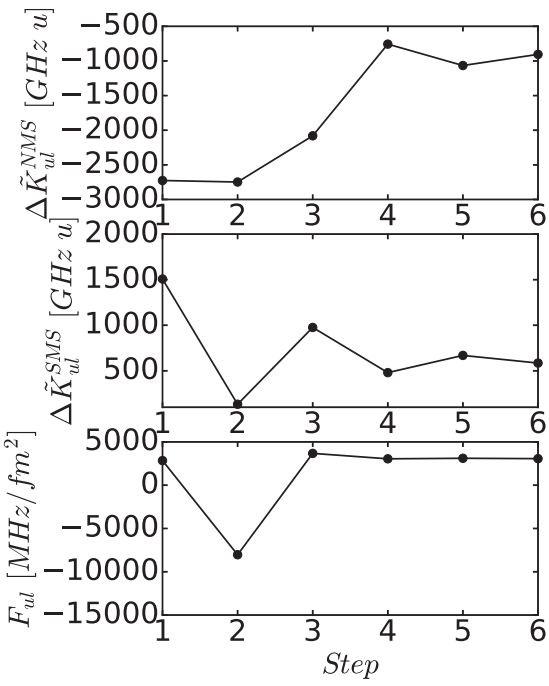

Fig. 1. Transition isotope shift electronic parameters, i.e. the normal mass shift parameter $\Delta \tilde{K}_{u l}^{R N M S}$, the specific mass shift parameter $\Delta \tilde{K}_{u l}^{R S M S}$ and the field-shift factor $F_{u l}$, for the transition $5 \mathrm{p}^{32} \mathrm{P}_{3 / 2}^{\mathrm{o}}-5 \mathrm{p}^{2} 6 \mathrm{~s}^{5} \mathrm{P}_{3 / 2}$ at $\lambda=363.8 \mathrm{~nm}$ in $\mathrm{Sb} \mathrm{I}$ as function of the MCDHF calculation step (Model A). For each parameter, a reasonable convergence has been achieved in the last three steps although residual oscillations are observed for the mass shift parameters.

the transition energies but has deteriorated the electronic isotope shift factors in contrast to a previous study in Mg I [27]. It is hard to have a good estimation of the relative importance of the different types of correlation on the electronic IS parameters from the previous analysis [26,27]. In fact, these studies all started from an VV calculation that accounted for a fair but still limited amount of VV correlation. It would be worthwhile to investigate the impact of the different nature of the FS and mass shift contributions that originate, respectively, from one- and two-body operators, as a function of the excitations considered beyond the mono- or multireference Dirac-Hartree-Fock model. Such an analysis cannot be done easily with the presently available multiconfiguration codes and is beyond the scope of the present work. Opening the $4 \mathrm{~d}$ subshell in Model B is justified by its closeness to the valence shells but opening the $4 \mathrm{~s}$ shell would be for example also needed for getting a better description of the change in electron density conditioning the FS. The present calculations are therefore undoubtedly incomplete while complete ones remain out of reach due to the complexity of a five valence electron atomic structure.

Concerning our model that produces the best results, i.e. Model $A$, the normal mass shift and specific mass shift parameters are relatively close to each other in absolute value but having opposite signs, they cancel each other, leading to the dominance of the field-shift effects over the total mass shift for most of the lines. In addition, the line field-shift factor changes sign, depending if the $5 p^{2} 6 s$ configuration is the upper or the lower configuration of the considered transition. The FS factor is indeed proportional to the change of the electron densities at the nucleus (see Eqs. (10) and (11)), and this density is definitely larger for the s orbitals than for the $\mathrm{p}$ ones.

Examples of convergence of the line IS electronic parameters are given in Figs. 1-3 for the three different types of transitions considered in this analysis, i.e. $5 p^{3}-5 p^{2} 6 s, 5 p^{2} 6 s-5 p^{2} 6 p$ and $5 p^{2} 6 s-5 p^{2} 7 p$ respectively. One can see in each figure that a reasonable convergence has been achieved in the last three steps of our calculations although some residual oscillations are observed for the mass shift parameters.

The so-called 'scaling law', where the normal mass shift is proportional to the line frequency in the non-relativistic limit $[21,22]$, has been used by Sobolewski et al. [5] to interpret their IS mea- 
Table 1

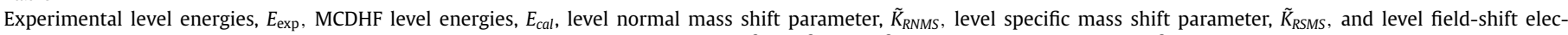

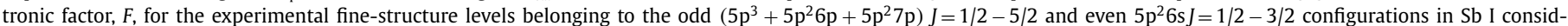
ered in this work.

\begin{tabular}{|c|c|c|c|c|c|c|}
\hline Designation $^{(a)}$ & $\begin{array}{l}E_{\exp }^{(a)} \\
\left(\mathrm{cm}^{-1}\right)\end{array}$ & $\begin{array}{l}E_{c a l} \\
\left(\mathrm{~cm}^{-1}\right)\end{array}$ & $\begin{array}{l}\Delta E^{(*)} \\
\left(\mathrm{cm}^{-1}\right)\end{array}$ & $\begin{array}{l}\tilde{K}_{R N M S} \\
(\mathrm{GHz} \mathrm{u})\end{array}$ & $\begin{array}{l}\tilde{K}_{R S M S} \\
(\mathrm{GHz} \mathrm{u})\end{array}$ & $\begin{array}{l}F \\
\left(\mathrm{GHz} \mathrm{fm}^{-2}\right)\end{array}$ \\
\hline \multirow[t]{2}{*}{$5 p^{3}{ }^{4} S_{3 / 2}^{o}$} & 0 & $0^{b}$ & $0^{b}$ & $2.2873356(+7)^{b}$ & $-6.7963360(+6)^{b}$ & $5.2308631(+4)^{b}$ \\
\hline & & $0^{c}$ & $0^{c}$ & $2.2871119(+7)^{c}$ & $-6.7919472(+6)^{c}$ & $5.2309407(+4)^{c}$ \\
\hline \multirow{2}{*}{$5 p^{3}{ }^{2} D_{3 / 2}^{o}$} & 8512 & $8873^{b}$ & $-361^{b}$ & $2.2873153(+7)^{b}$ & $-6.7962419(+6)^{b}$ & $5.2308758(+4)^{b}$ \\
\hline & & $9191^{c}$ & $-678^{c}$ & $2.2871014(+7)^{c}$ & $-6.7920006(+6)^{c}$ & $5.2309478(+4)^{c}$ \\
\hline \multirow{2}{*}{$5 p^{3}{ }^{2} D_{5 / 2}^{o}$} & 9854 & $10097^{b}$ & $-243^{b}$ & $2.2873049(+7)^{b}$ & $-6.7961486(+6)^{b}$ & $5.2308713(+4)^{b}$ \\
\hline & & $10898^{c}$ & $-1044^{c}$ & $2.2883982(+7)^{c}$ & $-6.7984042(+6)^{c}$ & $5.2310564(+4)^{c}$ \\
\hline \multirow{2}{*}{$5 \mathrm{p}^{3}{ }^{2} \mathrm{P}_{1 / 2}^{o}$} & 16395 & $16707^{b}$ & $-312^{b}$ & $2.2872936(+7)^{b}$ & $-6.7961034(+6)^{b}$ & $5.2308648(+4)^{b}$ \\
\hline & & $16786^{c}$ & $-391^{c}$ & $2.2870777(+7)^{c}$ & $-6.7918858(+6)^{c}$ & $5.2309274(+4)^{c}$ \\
\hline \multirow[t]{2}{*}{$5 \mathrm{p}^{3}{ }^{2} \mathrm{P}_{3 / 2}^{o}$} & 18464 & $18519^{b}$ & $-55^{b}$ & $2.2872774(+7)^{b}$ & $-6.7959604(+6)^{b}$ & $5.2308551(+4)^{b}$ \\
\hline & & $18229^{c}$ & $235^{c}$ & $2.2870682(+7)^{c}$ & $-6.7918483(+6)^{c}$ & $5.2309194(+4)^{c}$ \\
\hline \multirow[t]{2}{*}{$5 p^{2}\left({ }^{3} P\right) 6 s{ }^{4} P_{1 / 2}$} & 43249 & $48140^{b}$ & $-4891^{b}$ & $2.2872034(+7)^{b}$ & $-6.7955270(+6)^{b}$ & $5.2311670(+4)^{b}$ \\
\hline & & $41827^{c}$ & $1422^{c}$ & $2.2870371(+7)^{c}$ & $-6.7915050(+6)^{c}$ & $5.2311834(+4)^{c}$ \\
\hline \multirow[t]{2}{*}{$5 \mathrm{p}^{2}\left({ }^{3} \mathrm{P}\right) 6 \mathrm{~s}{ }^{4} \mathrm{P}_{3 / 2}$} & 45945 & $50381^{b}$ & $-4436^{b}$ & $2.2871871(+7)^{b}$ & $-6.7953766(+6)^{b}$ & $5.2311611(+4)^{b}$ \\
\hline & & $45282^{c}$ & $664^{c}$ & $2.2870445(+7)^{c}$ & $-6.7918324(+6)^{c}$ & $5.2311872(+4)^{c}$ \\
\hline \multirow[t]{2}{*}{$5 \mathrm{p}^{2}\left({ }^{3} \mathrm{P}\right) 6 \mathrm{~s}{ }^{2} \mathrm{P}_{1 / 2}$} & 46991 & $51886^{b}$ & $-4895^{b}$ & $2.2871804(+7)^{b}$ & $-6.7951891(+6)^{b}$ & $5.2311425(+4)^{b}$ \\
\hline & & $46063^{c}$ & $928^{c}$ & $2.2870347(+7)^{c}$ & $-6.7915527(+6)^{c}$ & $5.2311737(+4)^{c}$ \\
\hline \multirow{2}{*}{$5 \mathrm{p}^{2}\left({ }^{3} \mathrm{P}\right) 6 \mathrm{~s}^{2} \mathrm{P}_{3 / 2}$} & 49391 & $54354^{b}$ & $-4963^{b}$ & $2.2871683(+7)^{b}$ & $-6.7951180(+6)^{b}$ & $5.2311403(+4)^{b}$ \\
\hline & & $48834^{c}$ & $557^{c}$ & $2.2870432(+7)^{c}$ & $-6.7916090(+6)^{c}$ & $5.2311696(+4)^{c}$ \\
\hline \multirow{2}{*}{$5 p^{2}\left({ }^{3} P_{0}\right) 6 p(0,1 / 2)_{1 / 2}^{o}$} & 51676 & $50875^{b}$ & $801^{b}$ & $2.2871753(+7)^{b}$ & $-6.7952973(+6)^{b}$ & $5.2310265(+4)^{b}$ \\
\hline & & $52039^{c}$ & $-362^{c}$ & $2.2870143(+7)^{c}$ & $-6.7920601(+6)^{c}$ & $5.2310913(+4)^{c}$ \\
\hline $5 \mathrm{p}^{2}\left({ }^{3} \mathrm{P}_{0}\right) 6 \mathrm{p}(0,3 / 2)_{3 / 2}^{o}$ & 52612 & $51778^{b}$ & $834^{b}$ & $2.2871734(+7)^{b}$ & $-6.7952748(+6)^{b}$ & $5.2310173(+4)^{b}$ \\
\hline & & $52937^{c}$ & $-324^{c}$ & $2.2870174(+7)^{c}$ & $-6.7920818(+6)^{c}$ & $5.2310908(+4)^{c}$ \\
\hline $5 p^{2}\left({ }^{3} P_{1}\right) 6 p(1,1 / 2)_{1 / 2}^{o}$ & 54196 & $53173^{b}$ & $1023^{b}$ & $2.2871555(+7)^{b}$ & $-6.7951226(+6)^{b}$ & $5.2310162(+4)^{b}$ \\
\hline & & $54722^{c}$ & $-525^{c}$ & $2.2870125(+7)^{c}$ & $-6.7922237(+6)^{c}$ & $5.2310863(+4)^{c}$ \\
\hline $5 \mathrm{p}^{2}\left({ }^{3} \mathrm{P}_{1}\right) 6 \mathrm{p}(1,1 / 2)_{3 / 2}^{o}$ & 55134 & $54125^{b}$ & $1009^{b}$ & $2.2871566(+7)^{b}$ & $-6.7951287(+6)^{b}$ & $5.2310256(+4)^{b}$ \\
\hline & & $55613^{c}$ & $-479^{c}$ & $2.2870166(+7)^{c}$ & $-6.7922280(+6)^{c}$ & $5.2310873(+4)^{c}$ \\
\hline $5 p^{2}\left({ }^{1} D\right) 6 s{ }^{2} D_{3 / 2}$ & 55233 & $58351^{b}$ & $-3118^{b}$ & $2.2871052(+7)^{b}$ & $-6.7958902(+6)^{b}$ & $5.2311519(+4)^{b}$ \\
\hline & & $55132^{c}$ & $101^{c}$ & $2.2870404(+7)^{c}$ & $-6.7917436(+6)^{c}$ & $5.2311724(+4)^{c}$ \\
\hline $5 p^{2}\left({ }^{3} P_{1}\right) 6 p(1,3 / 2)_{5 / 2}^{o}$ & 55252 & $54218^{b}$ & $1034^{b}$ & $2.2871544(+7)^{b}$ & $-6.7951064(+6)^{b}$ & $5.2310155(+4)^{b}$ \\
\hline & & $56009^{c}$ & $-757^{c}$ & $2.2874074(+7)^{c}$ & $-6.7943551(+6)^{c}$ & $5.2310640(+4)^{c}$ \\
\hline $5 \mathrm{p}^{2}\left({ }^{3} \mathrm{P}_{1}\right) 6 \mathrm{p}(1,3 / 2)_{3 / 2}^{0}$ & 55864 & $54829^{b}$ & $1035^{b}$ & $2.2871549(+7)^{b}$ & $-6.7951165(+6)^{b}$ & $5.2310159(+4)^{b}$ \\
\hline & & $56224^{c}$ & $-360^{c}$ & $2.2870165(+7)^{c}$ & $-6.7922179(+6)^{c}$ & $5.2310858(+4)^{c}$ \\
\hline $5 p^{2}\left({ }^{3} P_{1}\right) 6 p(1,3 / 2)_{1 / 2}^{o}$ & 55993 & $54953^{b}$ & $1040^{b}$ & $2.2871538(+7)^{b}$ & $-6.7951078(+6)^{b}$ & $5.2310179(+4)^{b}$ \\
\hline & & $56403^{c}$ & $-409^{c}$ & $2.2870167(+7)^{c}$ & $-6.7922348(+6)^{c}$ & $5.2310861(+4)^{c}$ \\
\hline $5 \mathrm{p}^{2}\left({ }^{3} \mathrm{P}_{2}\right) 6 \mathrm{p}(2,1 / 2)_{5 / 2}^{0}$ & 57410 & $56362^{b}$ & $1048^{b}$ & $2.2871453(+7)^{b}$ & $-6.7950431(+6)^{b}$ & $5.2310142(+4)^{b}$ \\
\hline & & $57964^{c}$ & $-554^{c}$ & $2.2875949(+7)^{c}$ & $-6.7940230(+6)^{c}$ & $5.2309202(+4)^{c}$ \\
\hline $5 \mathrm{p}^{2}\left({ }^{3} \mathrm{P}_{2}\right) 6 \mathrm{p}(2,1 / 2)_{3 / 2}^{0}$ & 58076 & $56952^{b}$ & $1124^{b}$ & $2.2871436(+7)^{b}$ & $-6.7950187(+6)^{b}$ & $5.2310164(+4)^{b}$ \\
\hline & & $58264^{c}$ & $-188^{c}$ & $2.2870188(+7)^{c}$ & $-6.7921998(+6)^{c}$ & $5.2310793(+4)^{c}$ \\
\hline $5 \mathrm{p}^{2}\left({ }^{3} \mathrm{P}_{2}\right) 6 \mathrm{p}(2,3 / 2)_{3 / 2}^{0}$ & 58589 & $57585^{b}$ & $1004^{b}$ & $2.2871462(+7)^{b}$ & $-6.7950615(+6)^{b}$ & $5.2310168(+4)^{b}$ \\
\hline & & $58979^{c}$ & $-390^{c}$ & $2.2870160(+7)^{c}$ & $-6.7922186(+6)^{c}$ & $5.2310833(+4)^{c}$ \\
\hline $5 \mathrm{p}^{2}\left({ }^{3} \mathrm{P}_{2}\right) 6 \mathrm{p}(2,3 / 2)_{1 / 2}^{o}$ & 58653 & $57605^{b}$ & $1048^{b}$ & $2.2871449(+7)^{b}$ & $-6.7950623(+6)^{b}$ & $5.2310131(+4)^{b}$ \\
\hline & & $58953^{c}$ & $-300^{c}$ & $2.2870161(+7)^{c}$ & $-6.7921988(+6)^{c}$ & $5.2310795(+4)^{c}$ \\
\hline $5 \mathrm{p}^{2}\left({ }^{3} \mathrm{P}_{2}\right) 6 \mathrm{p}(2,3 / 2)_{5 / 2}^{0}$ & 58835 & $57735^{b}$ & $1100^{b}$ & $2.2871426(+7)^{b}$ & $-6.7950169(+6)^{b}$ & $5.2310242(+4)^{b}$ \\
\hline & & $59420^{c}$ & $-585^{c}$ & $2.2878322(+7)^{c}$ & $-6.7942551(+6)^{c}$ & $5.2310620(+4)^{c}$ \\
\hline $5 p^{2}\left({ }^{3} P_{0}\right) 7 p(0,1 / 2)_{1 / 2}^{o}$ & 60765 & $60046^{b}$ & $719^{b}$ & $2.2871555(+7)^{b}$ & $-6.7952343(+6)^{b}$ & $5.2310791(+4)^{b}$ \\
\hline & & $61000^{c}$ & $-235^{c}$ & $2.2870031(+7)^{c}$ & $-6.7920630(+6)^{c}$ & $5.2310974(+4)^{c}$ \\
\hline $5 p^{2}\left({ }^{3} P_{0}\right) 7 p(0,3 / 2)_{3 / 2}^{o}$ & 61000 & $62672^{b}$ & $-1672^{b}$ & $2.2871363(+7)^{b}$ & $-6.7950657(+6)^{b}$ & $5.2310339(+4)^{b}$ \\
\hline & & $61133^{c}$ & $-133^{c}$ & $2.2870026(+7)^{c}$ & $-6.7920305(+6)^{c}$ & $5.2310964(+4)^{c}$ \\
\hline $5 \mathrm{p}^{2}\left({ }^{3} \mathrm{P}_{1}\right) 7 \mathrm{p}(1,1 / 2)_{1 / 2}^{0}$ & 63606 & $62743^{b}$ & $863^{b}$ & $2.2871327(+7)^{b}$ & $-6.7950290(+6)^{b}$ & $5.2310711(+4)^{b}$ \\
\hline & & $63987^{c}$ & $-381^{c}$ & $2.2869998(+7)^{c}$ & $-6.7922121(+6)^{c}$ & $5.2310943(+4)^{c}$ \\
\hline $5 p^{2}\left({ }^{3} P_{1}\right) 7 p(1,3 / 2)_{5 / 2}^{o}$ & 63791 & $62652^{b}$ & $1139^{b}$ & $2.2871336(+7)^{b}$ & $-6.7950437(+6)^{b}$ & $5.2310127(+4)^{b}$ \\
\hline & & $64399^{c}$ & $-608^{c}$ & $2.2870785(+7)^{c}$ & $-6.7935823(+6)^{c}$ & $5.2310206(+4)^{c}$ \\
\hline $5 p^{2}\left({ }^{3} P_{1}\right) 7 p(1,1 / 2)_{3 / 2}^{o}$ & 63798 & $63891^{b}$ & $-93^{b}$ & $2.2871233(+7)^{b}$ & $-6.7948852(+6)^{b}$ & $5.2310706(+4)^{b}$ \\
\hline & & $64124^{c}$ & $-325^{c}$ & $2.2870018(+7)^{c}$ & $-6.7921930(+6)^{c}$ & $5.2310926(+4)^{c}$ \\
\hline $5 \mathrm{p}^{2}\left({ }^{3} \mathrm{P}_{1}\right) 7 \mathrm{p}(1,3 / 2)_{3 / 2}^{o}$ & 63901 & $64864^{b}$ & $-963^{b}$ & $2.2871195(+7)^{b}$ & $-6.7948981(+6)^{b}$ & $5.2310686(+4)^{b}$ \\
\hline & & $64162^{c}$ & $-261^{c}$ & $2.2870023(+7)^{c}$ & $-6.7922031(+6)^{c}$ & $5.2310911(+4)^{c}$ \\
\hline $5 p^{2}\left({ }^{3} P_{1}\right) 7 p(1,3 / 2)_{1 / 2}^{o}$ & 64209 & $65299^{b}$ & $-1090^{b}$ & $2.2871242(+7)^{b}$ & $-6.7949665(+6)^{b}$ & $5.2310701(+4)^{b}$ \\
\hline & & $64434^{c}$ & $-225^{c}$ & $2.2870036(+7)^{c}$ & $-6.7922071(+6)^{c}$ & $5.2310923(+4)^{c}$ \\
\hline $5 p^{2}\left({ }^{1} D_{2}\right) 6 p(2,1 / 2)_{5 / 2}^{0}$ & 64512 & $63429^{b}$ & $1083^{b}$ & $2.2871189(+7)^{b}$ & $-6.7948527(+6)^{b}$ & $5.2310587(+4)^{b}$ \\
\hline & & $65230^{c}$ & $-717^{c}$ & $2.2881392(+7)^{c}$ & $-6.7944106(+6)^{c}$ & $5.2309917(+4)^{c}$ \\
\hline $5 \mathrm{p}^{2}\left({ }^{1} \mathrm{D}_{2}\right) 6 \mathrm{p}(2,3 / 2)_{5 / 2}^{0}$ & 64879 & $63818^{b}$ & $1079^{b}$ & $2.2871182(+7)^{b}$ & $-6.7948467(+6)^{b}$ & $5.2310597(+4)^{b}$ \\
\hline & & $65357^{c}$ & $-478^{c}$ & $2.2882873(+7)^{c}$ & $-6.7944702(+6)^{c}$ & $5.2309681(+4)^{c}$ \\
\hline $5 \mathrm{p}^{2}\left({ }^{1} \mathrm{D}_{2}\right) 6 \mathrm{p}(2,1 / 2)_{3 / 2}^{o}$ & 64985 & $65995^{b}$ & $-1010^{b}$ & $2.2871252(+7)^{b}$ & $-6.7949339(+6)^{b}$ & $5.2310581(+4)^{b}$ \\
\hline & & $65213^{c}$ & $-228^{c}$ & $2.2870105(+7)^{c}$ & $-6.7921793(+6)^{c}$ & $5.2310785(+4)^{c}$ \\
\hline $5 \mathrm{p}^{2}\left({ }^{1} \mathrm{D}_{2}\right) 6 \mathrm{p}(2,3 / 2)_{1 / 2}^{o}$ & 65480 & $64432^{b}$ & $1048^{b}$ & $2.2871195(+7)^{b}$ & $-6.7952343(+6)^{b}$ & $5.2310625(+4)^{b}$ \\
\hline & & $65756^{c}$ & $-276^{c}$ & $2.2870021(+7)^{c}$ & $-6.7921720(+6)^{c}$ & $5.2310818(+4)^{c}$ \\
\hline $5 \mathrm{p}^{2}\left({ }^{1} \mathrm{D}_{2}\right) 6 \mathrm{p}(2,3 / 2)_{3 / 2}^{0}$ & 65959 & $69551^{b}$ & $-3592^{b}$ & $2.2871059(+7)^{b}$ & $-6.7950047(+6)^{b}$ & $5.2310620(+4)^{b}$ \\
\hline & & $66068^{c}$ & $-109^{c}$ & $2.2870039(+7)^{c}$ & $-6.7921569(+6)^{c}$ & $5.2310813(+4)^{c}$ \\
\hline $5 p^{2}\left({ }^{3} P_{2}\right) 7 p(2,1 / 2)_{5 / 2}^{o}$ & 66362 & $66919^{b}$ & $-557^{b}$ & $2.2871244(+7)^{b}$ & $-6.7949727(+6)^{b}$ & $5.2310677(+4)^{b}$ \\
\hline & & $66631^{c}$ & $-269^{c}$ & $2.2871581(+7)^{c}$ & $-6.7941114(+6)^{c}$ & $5.2309074(+4)^{c}$ \\
\hline
\end{tabular}


Table 1 (continued)

\begin{tabular}{|c|c|c|c|c|c|c|}
\hline Designation $^{(a)}$ & $\begin{array}{l}E_{\exp }^{(a)} \\
\left(\mathrm{cm}^{-1}\right)\end{array}$ & $\begin{array}{l}E_{\text {cal }} \\
\left(\mathrm{cm}^{-1}\right)\end{array}$ & $\begin{array}{l}\Delta E^{(*)} \\
\left(\mathrm{cm}^{-1}\right)\end{array}$ & $\begin{array}{l}\tilde{K}_{R N M S} \\
(\mathrm{GHz} \mathrm{u})\end{array}$ & $\begin{array}{l}\tilde{K}_{R S M S} \\
(\mathrm{GHz} \mathrm{u})\end{array}$ & $\begin{array}{l}F \\
\left(\mathrm{GHz} \mathrm{fm}^{-2}\right)\end{array}$ \\
\hline \multirow[t]{2}{*}{$5 \mathrm{p}^{2}\left({ }^{3} \mathrm{P}_{2}\right) 7 \mathrm{p}(2,1 / 2)_{3 / 2}^{0}$} & 66542 & $72075^{b}$ & $5533^{b}$ & $2.2870933(+7)^{b}$ & $-6.7949083(+6)^{b}$ & $5.2310662(+4)^{b}$ \\
\hline & & $66441^{c}$ & $101^{c}$ & $2.2870033(+7)^{c}$ & $-6.7921193(+6)^{c}$ & $5.2310843(+4)^{c}$ \\
\hline \multirow[t]{2}{*}{$5 \mathrm{p}^{2}\left({ }^{3} \mathrm{P}_{2}\right) 7 \mathrm{p}(2,3 / 2)_{1 / 2}^{o}$} & 66685 & $65688^{b}$ & $997^{b}$ & $2.2871265(+7)^{b}$ & $-6.7949613(+6)^{b}$ & $5.2310644(+4)^{b}$ \\
\hline & & $66889^{c}$ & $-204^{c}$ & $2.2870064(+7)^{c}$ & $-6.7921392(+6)^{c}$ & $5.2310823(+4)^{c}$ \\
\hline \multirow[t]{2}{*}{$5 p^{2}\left({ }^{3} P_{2}\right) 7 p(2,3 / 2)_{5 / 2}^{o}$} & 66838 & $69551^{b}$ & $-2713^{b}$ & $2.2871260(+7)^{b}$ & $-6.7949710(+6)^{b}$ & $5.2310658(+4)^{b}$ \\
\hline & & $67080^{c}$ & $-243^{c}$ & $2.2870846(+7)^{c}$ & $-6.7939822(+6)^{c}$ & $5.2309081(+4)^{c}$ \\
\hline \multirow[t]{2}{*}{$5 p^{2}\left({ }^{3} P_{2}\right) 7 p(2,3 / 2)_{3 / 2}^{o}$} & 66958 & $72125^{b}$ & $-5167^{b}$ & $2.2870936(+7)^{b}$ & $-6.7949083(+6)^{b}$ & $5.2310240(+4)^{b}$ \\
\hline & & $67173^{c}$ & $-215^{c}$ & $2.2870086(+7)^{c}$ & $-6.7921515(+6)^{c}$ & $5.2310803(+4)^{c}$ \\
\hline
\end{tabular}

(a) Hassini et al. [25] as taken from NIST [6]. (b) This work: Model A. (c) This work: Model B. $\left(^{*}\right) \Delta E=E_{\exp }-E_{\text {cal }}$.

Table 2

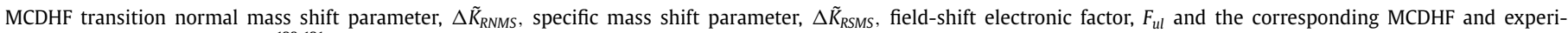
mental total isotope shifts, $\delta v_{u l}^{123,121}$, for the E1 transitions measured by Buchholz et al. [4] and Sobolewski et al. [5] between the two stable isotopes of Sb I.

\begin{tabular}{|c|c|c|c|c|c|c|c|}
\hline \multirow{2}{*}{$\begin{array}{l}\lambda^{a} \\
(\mathrm{~nm})\end{array}$} & \multirow[t]{2}{*}{ Transition $^{a}$} & \multirow{2}{*}{$\begin{array}{l}\Delta \tilde{K}_{R N M S}^{b} \\
(\mathrm{GHz} \mathrm{u})\end{array}$} & \multirow{2}{*}{$\begin{array}{l}\Delta \tilde{K}_{R S M S}^{b} \\
(\mathrm{GHz} \mathrm{u})\end{array}$} & \multirow{2}{*}{$\begin{array}{l}F_{u l}{ }^{b} \\
\left(\mathrm{MHz} / \mathrm{fm}^{2}\right)\end{array}$} & \multicolumn{3}{|c|}{$\delta v_{u l}^{123,121}(\mathrm{MHz})$} \\
\hline & & & & & $\mathrm{MCDHF}^{b}$ & $\mathrm{BUCH}^{d}$ & $\mathrm{SOBO}^{e}$ \\
\hline \multirow[t]{2}{*}{252.9} & \multirow{2}{*}{$5 p^{32} D_{5 / 2}^{o}-5 p^{2}\left({ }^{3} P\right) 6 s^{2} P_{3 / 2}$} & $-1369^{b}$ & $1032^{b}$ & $2689^{b}$ & $239^{b}$ & $96 \pm 21$ & \\
\hline & & $-13551^{c}$ & $6795^{c}$ & $1132^{c}$ & $991^{c}$ & & \\
\hline \multirow[t]{2}{*}{259.8} & \multirow[t]{2}{*}{$5 p^{32} D_{3 / 2}^{o}-5 p^{2}\left({ }^{3} P\right) 6 s^{2} P_{1 / 2}$} & $-1352^{b}$ & $1055^{b}$ & $2676^{b}$ & $233^{b}$ & $126 \pm 21$ & \\
\hline & & $-667^{c}$ & $448^{c}$ & $2259^{c}$ & $192^{c}$ & & \\
\hline \multirow[t]{2}{*}{277.0} & \multirow[t]{2}{*}{$5 p^{32} D_{5 / 2}^{o}-5 p^{2}\left({ }^{3} P\right) 6 s^{4} P_{3 / 2}$} & $-1181^{b}$ & $773^{b}$ & $2884^{b}$ & $263^{b}$ & $96 \pm 60$ & \\
\hline & & $-13537^{c}$ & $6572^{c}$ & $1308^{c}$ & $1032^{c}$ & & \\
\hline \multirow[t]{2}{*}{303.0} & \multirow{2}{*}{$5 \mathrm{p}^{32} \mathrm{P}_{1 / 2}^{\mathrm{o}}-5 \mathrm{p}^{2}\left({ }^{3} \mathrm{P}\right) 6 \mathrm{~s}^{2} \mathrm{P}_{3 / 2}$} & $-1255^{b}$ & $987^{b}$ & $2745^{b}$ & $235^{b}$ & $99 \pm 18$ & \\
\hline & & $-345^{c}$ & $277^{c}$ & $2422^{c}$ & $190^{c}$ & & \\
\hline \multirow[t]{2}{*}{326.8} & \multirow{2}{*}{$5 \mathrm{p}^{32} \mathrm{P}_{1 / 2}^{\mathrm{o}}-5 \mathrm{p}^{2}\left({ }^{3} \mathrm{P}\right) 6 \mathrm{~s}^{2} \mathrm{P}_{1 / 2}$} & $-1134^{b}$ & $916^{b}$ & $2786^{b}$ & $230^{b}$ & $108 \pm 15$ & \\
\hline & & $-430^{c}$ & $333^{c}$ & $2462^{c}$ & $191^{c}$ & & \\
\hline \multirow[t]{2}{*}{363.8} & \multirow{2}{*}{$5 \mathrm{p}^{32} \mathrm{P}_{3 / 2}^{0}-5 \mathrm{p}^{2}\left({ }^{3} \mathrm{P}\right) 6 \mathrm{~s}^{4} \mathrm{P}_{3 / 2}$} & $-905^{b}$ & $585^{b}$ & $3064^{b}$ & $265^{b}$ & $150 \pm 30$ & $254 \pm 28$ \\
\hline & & $-237^{c}$ & $16^{c}$ & $2678^{c}$ & $223^{c}$ & & \\
\hline \multirow[t]{2}{*}{372.3} & \multirow[t]{2}{*}{$5 \mathrm{p}^{32} \mathrm{P}_{1 / 2}^{\mathrm{o}}-5 \mathrm{p}^{2}\left({ }^{3} \mathrm{P}\right) 6 \mathrm{~s}^{4} \mathrm{P}_{1 / 2}$} & $-903^{b}$ & $577^{b}$ & $3022^{b}$ & $262^{b}$ & $210 \pm 60$ & $255 \pm 10$ \\
\hline & & $-406^{c}$ & $381^{c}$ & $2560^{c}$ & $188^{c}$ & & \\
\hline \multirow[t]{2}{*}{403.4} & \multirow[t]{2}{*}{$5 \mathrm{p}^{32} \mathrm{P}_{3 / 2}^{\mathrm{o}}-5 \mathrm{p}^{2}\left({ }^{3} \mathrm{P}\right) 6 \mathrm{~s}^{4} \mathrm{P}_{1 / 2}$} & $-741^{b}$ & $434^{b}$ & $3119^{b}$ & $267^{b}$ & $180 \pm 45$ & $248 \pm 19$ \\
\hline & & $-311^{c}$ & $343^{c}$ & $2640^{c}$ & $186^{c}$ & & \\
\hline \multirow[t]{2}{*}{563.2} & \multirow[t]{2}{*}{$5 p^{2}\left({ }^{3} P\right) 6 s^{4} P_{1 / 2}-5 p^{2}\left({ }^{3} P_{0}\right) 7 p(0,3 / 2)_{3 / 2}^{o}$} & $-480^{b}$ & $293^{b}$ & $-1331^{b}$ & $-71^{b}$ & & $-80 \pm 17$ \\
\hline & & $-345^{c}$ & $-526^{c}$ & $-871^{c}$ & $54^{c}$ & & \\
\hline \multirow[t]{2}{*}{792.5} & \multirow{2}{*}{$5 \mathrm{p}^{2}\left({ }^{3} \mathrm{P}\right) 6 \mathrm{~s}^{4} \mathrm{P}_{1 / 2}-5 \mathrm{p}^{2}\left({ }^{3} \mathrm{P}_{1}\right) 6 \mathrm{p}(1,3 / 2)_{3 / 2}^{0}$} & $-486^{b}$ & $411^{b}$ & $-1511^{b}$ & $-99^{b}$ & & $-112 \pm 17$ \\
\hline & & $-206^{c}$ & $-713^{c}$ & $-976^{c}$ & $53^{c}$ & & \\
\hline \multirow[t]{2}{*}{841.2} & \multirow[t]{2}{*}{$5 p^{2}\left({ }^{3} P\right) 6 s^{4} P_{1 / 2}-5 p^{2}\left({ }^{3} P_{1}\right) 6 p(1,1 / 2)_{3 / 2}^{o}$} & $-301^{b}$ & $252^{b}$ & $-1414^{b}$ & $-96^{b}$ & & $-110 \pm 19$ \\
\hline & & $-205^{c}$ & $-723^{c}$ & $-961^{c}$ & $56^{c}$ & & \\
\hline \multirow[t]{2}{*}{1067.7} & \multirow[t]{2}{*}{$5 p^{2}\left({ }^{3} P\right) 6 s^{4} P_{1 / 2}-5 p^{2}\left({ }^{3} P_{0}\right) 6 p(0,3 / 2)_{3 / 2}^{0}$} & $-469^{b}$ & $399^{b}$ & $-1497^{b}$ & $-98^{b}$ & & $-116 \pm 14$ \\
\hline & & $-197^{c}$ & $-577^{c}$ & $-926^{c}$ & $37^{c}$ & & \\
\hline \multirow[t]{2}{*}{1074.2} & $5 p^{2}\left({ }^{3} P\right) 6 s^{4} P_{3 / 2}-5 p^{2}\left({ }^{3} P_{1}\right) 6 p(1,3 / 2)_{5 / 2}^{o}$ & $-418^{b}$ & $334^{b}$ & $-1456^{b}$ & $-94^{b}$ & & $-65 \pm 22$ \\
\hline & & $3629^{c}$ & $-2523^{c}$ & $-1232^{c}$ & $-238^{c}$ & & \\
\hline
\end{tabular}

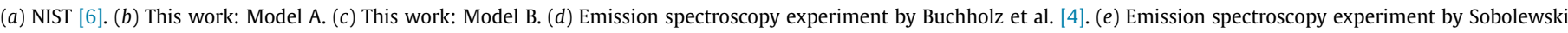
et al. [5].

Table 3

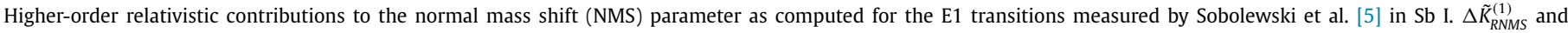

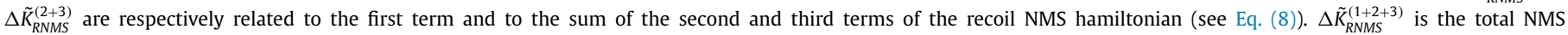
parameter.

\begin{tabular}{|c|c|c|c|c|}
\hline $\begin{array}{l}\lambda^{a} \\
(\mathrm{~nm})\end{array}$ & Transition $^{a}$ & $\begin{array}{c}\Delta \tilde{K}_{R N M S}^{(1) b} \\
(\mathrm{GHz} \mathrm{u})\end{array}$ & $\begin{array}{l}\Delta \tilde{K}_{R N M S}^{(2+3) b} \\
(\mathrm{GHz} \mathrm{u})\end{array}$ & $\begin{array}{l}\Delta \tilde{K}_{R N M S}^{(1+2+3) b} \\
(\mathrm{GHz} \mathrm{u})\end{array}$ \\
\hline 363.8 & $5 \mathrm{p}^{32} \mathrm{P}_{3 / 2}^{0}-5 \mathrm{p}^{2}\left({ }^{3} \mathrm{P}\right) 6 \mathrm{~s}^{4} \mathrm{P}_{3 / 2}$ & -539 & -366 & -905 \\
\hline 372.3 & $5 \mathrm{p}^{32} \mathrm{P}_{1 / 2}^{\mathrm{o}}-5 \mathrm{p}^{2}\left({ }^{3} \mathrm{P}\right) 6 \mathrm{~s}^{4} \mathrm{P}_{1 / 2}$ & -546 & -357 & -903 \\
\hline 403.4 & $5 \mathrm{p}^{32} \mathrm{P}_{3 / 2}^{\mathrm{o}}-5 \mathrm{p}^{2}\left({ }^{3} \mathrm{P}\right) 6 \mathrm{~s}^{4} \mathrm{P}_{1 / 2}$ & -257 & -484 & -741 \\
\hline 563.2 & $5 p^{2}\left({ }^{3} P\right) 6 s^{4} P_{1 / 2}-5 p^{2}\left({ }^{3} P_{0}\right) 7 p(0,3 / 2)_{3 / 2}^{0}$ & -568 & 88 & -480 \\
\hline 792.5 & $5 p^{2}\left({ }^{3} P\right) 6 s^{4} P_{1 / 2}-5 p^{2}\left({ }^{3} P_{1}\right) 6 p(1,3 / 2)_{3 / 2}^{o}$ & -719 & 233 & -486 \\
\hline 841.2 & $5 p^{2}\left({ }^{3} P\right) 6 s^{4} P_{1 / 2}-5 p^{2}\left({ }^{3} P_{1}\right) 6 p(1,1 / 2)_{3 / 2}^{o}$ & -398 & 97 & -301 \\
\hline 1067.7 & $5 p^{2}\left({ }^{3} P\right) 6 s^{4} P_{1 / 2}-5 p^{2}\left({ }^{3} P_{0}\right) 6 p(0,3 / 2)_{3 / 2}^{o}$ & -688 & 219 & -469 \\
\hline 1074.2 & $5 p^{2}\left({ }^{3} P\right) 6 s^{4} P_{3 / 2}-5 p^{2}\left({ }^{3} P_{1}\right) 6 p(1,3 / 2)_{5 / 2}^{0}$ & -574 & 156 & -418 \\
\hline
\end{tabular}

(a) NIST [6]. (b) This work: Model A.

surements for the normal mass shift (NMS) contribution. In Fig. 4, a plot of our MCDHF RNMS as calculated between the two stable ${ }^{123,121} \mathrm{Sb}$ isotopes as function of the line frequency is presented for the E1 transitions measured by Sobolewski et al. [5]. These values are compared to the scaling law. The latter is clearly broken down for this intermediate $Z$ element $(Z=51)$. Higher-order rel- ativistic contributions, $\Delta \tilde{K}_{R N M S}^{(2+3)}$, to the total RNMS parameter are shown in Table 3 for these lines where one can see that these contributions are far from being negligible. In addition, one also notice from this table that the term $\Delta \tilde{K}_{R N M S}^{(1)}$ related to the recoil operator $\frac{1}{M} \sum_{i} p_{i}^{2}$ (see Eq. (8)) is not inversely proportional to the transition wavelength in agreement with the statement that, as long as Dirac 
Table 4

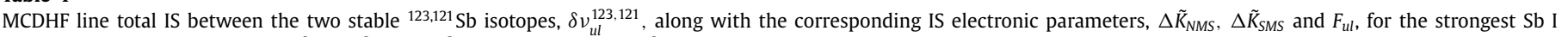
E1 transitions as classified as $\left\{5 p^{3}+5 p^{2} 6 p+5 p^{2} 7 p\right\} J=1 / 2-5 / 2-5 p^{2} 6 s J=1 / 2-3 / 2$ in the NIST database [6].

\begin{tabular}{|c|c|c|c|c|c|c|}
\hline $\begin{array}{l}\lambda^{a} \\
(\mathrm{~nm})\end{array}$ & Lower Level $^{a}$ & Upper Level $^{a}$ & $\begin{array}{l}\delta v_{u l}^{123,121 b} \\
(\mathrm{MHz})\end{array}$ & $\begin{array}{l}\Delta \tilde{K}_{N M S}^{b} \\
(\mathrm{GHz} \mathrm{u})\end{array}$ & $\begin{array}{l}\Delta \tilde{K}_{S M S}^{b} \\
(\mathrm{GHz} \mathrm{u})\end{array}$ & $\begin{array}{l}F_{u l}^{b} \\
\left(\mathrm{MHz} \mathrm{fm}^{-2}\right)\end{array}$ \\
\hline 202.4 & $5 \mathrm{p}^{3{ }^{4}} S_{3 / 2}^{o}$ & $5 \mathrm{p}^{2}\left({ }^{3} \mathrm{P}\right) 6 \mathrm{~s}^{2} \mathrm{P}_{3 / 2}$ & 262 & -1676 & 1220 & 2772 \\
\hline 212.7 & $5 p^{34} S_{3 / 2}^{o}$ & $5 \mathrm{p}^{2}\left({ }^{3} \mathrm{P}\right) 6 \mathrm{~s}^{2} \mathrm{P}_{1 / 2}$ & 257 & -1555 & 1149 & 2794 \\
\hline 217.6 & $5 \mathrm{p}^{34} \mathrm{~S}_{3 / 2}^{0}$ & $5 \mathrm{p}^{2}\left({ }^{3} \mathrm{P}\right) 6 \mathrm{~s}{ }^{4} \mathrm{P}_{3 / 2}$ & 287 & -1488 & 961 & 2980 \\
\hline 231.1 & $5 p^{34} S_{3 / 2}^{o}$ & $5 \mathrm{p}^{2}\left({ }^{3} \mathrm{P}\right) 6 \mathrm{~s}^{4} \mathrm{P}_{1 / 2}$ & 289 & -1324 & 810 & 3039 \\
\hline 244.5 & $5 \mathrm{p}^{32} \mathrm{D}_{3 / 2}^{o}$ & $5 \mathrm{p}^{2}\left({ }^{3} \mathrm{P}\right) 6 \mathrm{~s}^{2} \mathrm{P}_{3 / 2}$ & 238 & -1473 & 1126 & 2645 \\
\hline 267.1 & $5 \mathrm{p}^{32} \mathrm{D}_{3 / 2}^{o}$ & $5 \mathrm{p}^{2}\left({ }^{3} \mathrm{P}\right) 6 \mathrm{~s}^{4} \mathrm{P}_{3 / 2}$ & 263 & -1284 & 867 & 2853 \\
\hline 287.8 & $5 \mathrm{p}^{32} \mathrm{D}_{3 / 2}^{o}$ & $5 \mathrm{p}^{2}\left({ }^{3} \mathrm{P}\right) 6 \mathrm{~s}^{4} \mathrm{P}_{1 / 2}$ & 265 & -1120 & 716 & 2912 \\
\hline 323.2 & $5 \mathrm{p}^{32} \mathrm{P}_{3 / 2}^{o}$ & $5 \mathrm{p}^{2}\left({ }^{3} \mathrm{P}\right) 6 \mathrm{~s}^{2} \mathrm{P}_{3 / 2}$ & 240 & -1093 & 844 & 2852 \\
\hline 338.3 & $5 \mathrm{p}^{32} \mathrm{P}_{1 / 2}^{o}$ & $5 p^{2}\left({ }^{3} P\right) 6 s{ }^{4} P_{3 / 2}$ & 259 & -1065 & 727 & 2963 \\
\hline 475.7 & $5 \mathrm{p}^{2}\left({ }^{3} \mathrm{P}\right) 6 \mathrm{~s}{ }^{4} \mathrm{P}_{3 / 2}$ & $5 \mathrm{p}^{2}\left({ }^{3} \mathrm{P}_{2}\right) 7 \mathrm{p}(2,3 / 2)_{3 / 2}^{o}$ & -36 & -935 & 468 & -1371 \\
\hline 555.6 & $5 \mathrm{p}^{2}\left({ }^{3} \mathrm{P}\right) 6 \mathrm{~s}^{2} \mathrm{P}_{1 / 2}$ & $5 \mathrm{p}^{2}\left({ }^{3} \mathrm{P}_{2}\right) 6 \mathrm{p}(2,1 / 2)_{3 / 2}^{o}$ & -48 & -610 & 291 & -1261 \\
\hline 560.2 & $5 \mathrm{p}^{2}\left({ }^{3} \mathrm{P}\right) 6 \mathrm{~s}{ }^{4} \mathrm{P}_{3 / 2}$ & $5 \mathrm{p}^{2}\left({ }^{3} \mathrm{P}_{1}\right) 7 \mathrm{p}(1,3 / 2)_{5 / 2}^{o}$ & -86 & -682 & 525 & -1484 \\
\hline 573.0 & $5 \mathrm{p}^{2}\left({ }^{3} \mathrm{P}\right) 6 \mathrm{~s}^{2} \mathrm{P}_{3 / 2}$ & $5 \mathrm{p}^{2}\left({ }^{3} \mathrm{P}_{2}\right) 7 \mathrm{p}(2,3 / 2)_{5 / 2}^{o}$ & -17 & -423 & -47 & -745 \\
\hline 661.1 & $5 \mathrm{p}^{2}\left({ }^{3} \mathrm{P}\right) 6 \mathrm{~s}^{2} \mathrm{P}_{3 / 2}$ & $5 \mathrm{p}^{2}\left({ }^{1} \mathrm{D}_{2}\right) 6 \mathrm{p}(2,1 / 2)_{5 / 2}^{o}$ & -28 & -494 & 265 & -816 \\
\hline 784.4 & $5 \mathrm{p}^{2}\left({ }^{3} \mathrm{P}\right) 6 \mathrm{~s}{ }^{4} \mathrm{P}_{1 / 2}$ & $5 \mathrm{p}^{2}\left({ }^{3} \mathrm{P}_{1}\right) 6 \mathrm{p}(1,3 / 2)_{1 / 2}^{o}$ & -98 & -497 & 420 & -1491 \\
\hline 857.3 & $5 \mathrm{p}^{2}\left({ }^{3} \mathrm{P}\right) 6 \mathrm{~s}^{2} \mathrm{P}_{1 / 2}$ & $5 \mathrm{p}^{2}\left({ }^{3} \mathrm{P}_{2}\right) 6 \mathrm{p}(2,3 / 2)_{1 / 2}^{o}$ & -65 & -35 & 139 & -1294 \\
\hline 861.9 & $5 \mathrm{p}^{2}\left({ }^{3} \mathrm{P}\right) 6 \mathrm{~s}^{2} \mathrm{P}_{1 / 2}$ & $5 \mathrm{p}^{2}\left({ }^{3} \mathrm{P}_{2}\right) 6 \mathrm{p}(2,3 / 2)_{3 / 2}^{o}$ & -62 & -342 & 127 & -1257 \\
\hline 913.2 & $5 \mathrm{p}^{2}\left({ }^{3} \mathrm{P}\right) 6 \mathrm{~s}{ }^{4} \mathrm{P}_{1 / 2}$ & $5 \mathrm{p}^{2}\left({ }^{3} \mathrm{P}_{1}\right) 6 \mathrm{p}(1,1 / 2)_{1 / 2}^{o}$ & -99 & -480 & 405 & -1508 \\
\hline 994.9 & $5 \mathrm{p}^{2}\left({ }^{3} \mathrm{P}\right) 6 \mathrm{~s}{ }^{4} \mathrm{P}_{3 / 2}$ & $5 \mathrm{p}^{2}\left({ }^{3} \mathrm{P}_{1}\right) 6 \mathrm{p}(1,3 / 2)_{1 / 2}^{o}$ & -95 & -333 & 269 & -1432 \\
\hline 1007.8 & $5 \mathrm{p}^{2}\left({ }^{3} \mathrm{P}\right) 6 \mathrm{~s}{ }^{4} \mathrm{P}_{3 / 2}$ & $5 \mathrm{p}^{2}\left({ }^{3} \mathrm{P}_{1}\right) 6 \mathrm{p}(1,3 / 2)_{3 / 2}^{o}$ & -96 & -321 & 260 & -1452 \\
\hline 1058.5 & $5 \mathrm{p}^{2}\left({ }^{3} \mathrm{P}\right) 6 \mathrm{~s}^{2} \mathrm{P}_{3 / 2}$ & $5 \mathrm{p}^{2}\left({ }^{3} \mathrm{P}_{2}\right) 6 \mathrm{p}(2,3 / 2)_{5 / 2}^{\circ}$ & -47 & -347 & 74 & -1161 \\
\hline 1079.4 & $5 \mathrm{p}^{2}\left({ }^{3} \mathrm{P}\right) 6 \mathrm{~s}^{2} \mathrm{P}_{3 / 2}$ & $5 \mathrm{p}^{2}\left({ }^{3} \mathrm{P}_{2}\right) 6 \mathrm{p}(2,3 / 2)_{1 / 2}^{o}$ & -70 & -234 & 67 & -1272 \\
\hline 1086.9 & $5 \mathrm{p}^{2}\left({ }^{3} \mathrm{P}\right) 6 \mathrm{~s}^{2} \mathrm{P}_{3 / 2}$ & $5 \mathrm{p}^{2}\left({ }^{3} \mathrm{P}_{2}\right) 6 \mathrm{p}(2,3 / 2)_{3 / 2}^{o}$ & -67 & -221 & 56 & -1235 \\
\hline 1088.0 & $5 \mathrm{p}^{2}\left({ }^{3} \mathrm{P}\right) 6 \mathrm{~s}{ }^{4} \mathrm{P}_{3 / 2}$ & $5 \mathrm{p}^{2}\left({ }^{3} \mathrm{P}_{1}\right) 6 \mathrm{p}(1,1 / 2)_{3 / 2}^{0}$ & -96 & -305 & 248 & -1355 \\
\hline 1110.5 & $5 \mathrm{p}^{2}\left({ }^{3} \mathrm{P}\right) 6 \mathrm{~s}^{2} \mathrm{P}_{1 / 2}$ & $5 \mathrm{p}^{2}\left({ }^{3} \mathrm{P}_{1}\right) 6 \mathrm{p}(1,3 / 2)_{1 / 2}^{o}$ & -65 & -266 & 81 & -1246 \\
\hline 1126.6 & $5 \mathrm{p}^{2}\left({ }^{3} \mathrm{P}\right) 6 \mathrm{~s}^{2} \mathrm{P}_{1 / 2}$ & $5 \mathrm{p}^{2}\left({ }^{3} \mathrm{P}_{1}\right) 6 \mathrm{p}(1,3 / 2)_{3 / 2}^{o}$ & -66 & -254 & 72 & -1266 \\
\hline 1186.3 & $5 \mathrm{p}^{2}\left({ }^{3} \mathrm{P}\right) 6 \mathrm{~s}{ }^{4} \mathrm{P}_{1 / 2}$ & $5 \mathrm{p}^{2}\left({ }^{3} \mathrm{P}_{0}\right) 6 \mathrm{p}(0,1 / 2)_{1 / 2}^{o}$ & -95 & -281 & 230 & -1405 \\
\hline 1211.6 & $5 \mathrm{p}^{2}\left({ }^{3} \mathrm{P}\right) 6 \mathrm{~s}{ }^{4} \mathrm{P}_{3 / 2}$ & $5 \mathrm{p}^{2}\left({ }^{3} \mathrm{P}_{1}\right) 6 \mathrm{p}(1,1 / 2)_{1 / 2}^{o}$ & -97 & -316 & 254 & -1449 \\
\hline 1227.7 & $5 \mathrm{p}^{2}\left({ }^{3} \mathrm{P}\right) 6 \mathrm{~s}^{2} \mathrm{P}_{1 / 2}$ & $5 \mathrm{p}^{2}\left({ }^{3} \mathrm{P}_{1}\right) 6 \mathrm{p}(1,1 / 2)_{3 / 2}^{o}$ & -66 & -238 & 60 & -1169 \\
\hline 1246.7 & $5 \mathrm{p}^{2}\left({ }^{3} \mathrm{P}\right) 6 \mathrm{~s}^{2} \mathrm{P}_{3 / 2}$ & $5 \mathrm{p}^{2}\left({ }^{3} \mathrm{P}_{2}\right) 6 \mathrm{p}(2,1 / 2)_{5 / 2}^{o}$ & -71 & -257 & 101 & -1261 \\
\hline
\end{tabular}

(a) NIST [6]. (b) This work: Model A.

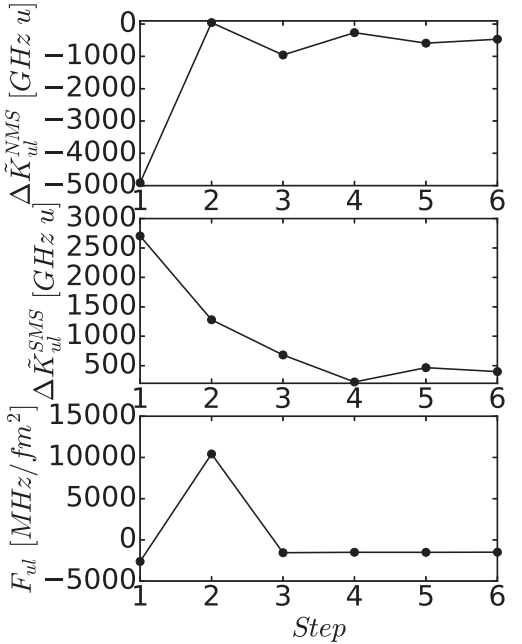

Fig. 2. Transition isotope shift electronic parameters, i.e. the normal mass shift parameter $\Delta \tilde{K}_{u l}^{R N S}$, the specific mass shift parameter $\Delta \tilde{K}_{u l}^{R S M S}$ and the field-shift factor $F_{u l}$, for the transition $5 \mathrm{p}^{2}\left({ }^{3} \mathrm{P}\right) 6 \mathrm{~s}^{4} \mathrm{P}_{1 / 2}-5 \mathrm{p}^{2}\left({ }^{3} \mathrm{P}_{0}\right) 6 \mathrm{p}(0,3 / 2)_{3 / 2}^{0}$ at $\lambda=1067.7 \mathrm{~nm}$ in Sb I as function of the MCDHF calculation step (Model A). For each parameter, a reasonable convergence has been achieved in the last three steps although residual oscillations are observed for the mass shift parameters.

electronic wave functions are used, one cannot expect that the operator $\frac{1}{M} \sum_{i} p_{i}^{2}$ leads to the scaling law $[21,23]$.

Finally, the MCDHF line total IS between the two stable ${ }^{123,121} \mathrm{Sb}$ isotopes, $\delta v_{u l}^{123,121}$, along with the corresponding IS electronic parameters are given in Table 4 for the strongest Sb I E1 transitions as classified as $\left\{5 p^{3}+5 p^{2} 6 p+\right.$

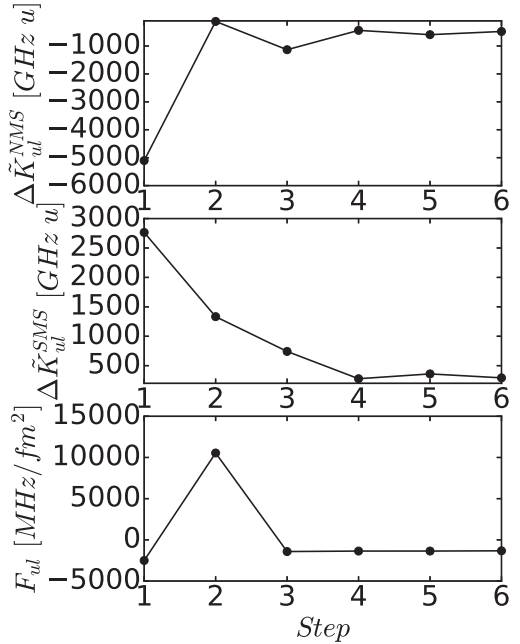

Fig. 3. Transition isotope shift electronic parameters, i.e. the normal mass shift parameter $\Delta \tilde{K}_{u l}^{R N M S}$, the specific mass shift parameter $\Delta \tilde{K}_{u l}^{R S S}$ and the field-shift factor $F_{u l}$, for the transition $5 \mathrm{p}^{2}\left({ }^{3} \mathrm{P}\right) 6 \mathrm{~s}^{4} \mathrm{P}_{1 / 2}-5 \mathrm{p}^{2}\left({ }^{3} \mathrm{P}_{0}\right) 7 \mathrm{p}(0,3 / 2)_{3 / 2}^{0}$ at $\lambda=563.2 \mathrm{~nm}$ in $\mathrm{Sb}$ I as function of the MCDHF calculation step (Model A). For each parameter, a reasonable convergence has been achieved in the last three steps although residual oscillations are observed for the mass shift parameters.

$\left.5 p^{2} 7 \mathrm{p}\right\} J=1 / 2-5 / 2-5 p^{2} 6 s \quad J=1 / 2-3 / 2$ in the NIST database [6] and not reported in Table 2. These complete the latter table and provide IS predictions and electronic parameters that will be relevant for both future atomic and nuclear physics investigations. 


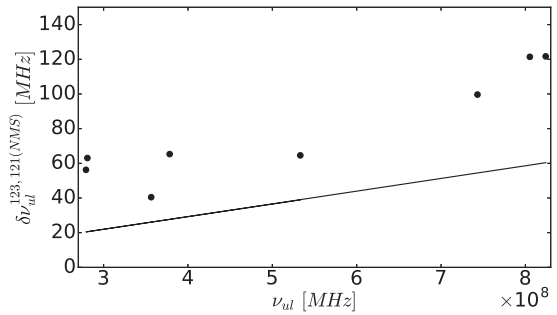

Fig. 4. Line normal mass shift, $\delta v_{u J}^{123,121(N M S)}$, between the two stable $\mathrm{Sb}$ isotopes as function of the transition frequency, $v_{u l}$. A comparison between our MCDHF model (Model A: black dots) and the scaling law, $\delta v_{u l}^{123,121(N M S)}=7.31886 \times 10^{-8} v_{u l}$, as used by Sobolewski et al. [5] (black line) is shown. The eight E1 transitions measured by Sobolewski et al. [5] (see Table 3) have been selected in this figure.

\section{Conclusions}

The fully relativistic electronic isotope shift parameters have been computed using the MCDHF ab initio method limited to valence correlation for the fine-structure levels belonging to the even $5 p^{2} 6 s J=1 / 2-3 / 2$ and odd $\left\{5 p^{3}+5 p^{2} 6 p+5 p^{2} 7 p\right\} J=1 / 2-5 / 2$ configurations of $\mathrm{Sb}$ I and for E1 transitions connecting both parities. These were used to determine the IS between the two stable ${ }^{123,121} \mathrm{Sb}$ isotopes for the E1 lines measured by Sobolewski et al. [5] and by Buchholz et al. [4]. They support the recent experimental values [5]. Although striking differences are seen with the older measurements [4], the large uncertainty affecting the change in mean-square nuclear charge radius between the two stable isotopes[3] does not allow to reject these experimental values. This illustrates the need of a more accurate determination of this nuclear parameter. $A b$ initio IS predictions are proposed for other 31 strong E1 transitions classified in the NIST database [6]. This new data set will be relevant for future studies in bothatomic and nuclear physics.

Adding more valence correlation by extending the multireference function and considering one hole in the $4 \mathrm{~d}$ subshell to take into account of some core-polarization improves systematically the excitation energies. More elaborate models involving excitations deeper in the core are needed to getIS parameters consistent with observation. Unfortunately the current status of GRASP2K [11] does not allow us to perform such calculations. The use of the partitioned correlation function interaction (PCFI) approach [28] may solve the problem of obtaining a converged MCDHF model beyond valence-valence correlation in Sb I. Tools for investigating the specific contributions from every excitation class would be welcome.

\section{Acknowledgments}

PP and PQ are, respectively, Research Associate and Research Director of the Belgian Fund for Scientific Research F.R.S.-FNRS. Financial support from this organization is gratefully acknowledged (grant nos. FC 49142, FC 37552 and CDR J.0047.16). This work has been funded by the Interuniversity Attraction Poles Programme initiated by the Belgian Policy Office (BriX network P7/12).

\section{References}

[1] Garcia Ruiz RF, Bissell ML, Blaum K, Ekström A, Frömmgen N, Hagen G, Hammen M, Hebeler K, Holt JD, Jansen GR, Kowalska M, Kreim K, Nazarewicz W, Neugart R, Neyens G, Nörtershäuser W, Papenbrock T, Papuga J, Schwenk A, Simonis J, Wendt KA, Yordanov DT. Unexpectedly large charge radii of neutron-rich calcium isotopes. Nat Phys 2016;12:594-8.
[2] Table of nuclides, Nuclear Data Center at KAERI (Korea Atomic Energy Research Institute); 2017. [http://www.atom.kaeri.re.kr/nuchart/ (last accessed February 17)].

[3] Angeli I, Marinova KP. Table of experimental nuclear ground state charge radii: an update. At Data Nucl Data Tables 2013;99:69-95.

[4] Buchholz B, Kronfeldt HD, Müller G, Voss M, Winkler R. Electric and magnetic hyperfinestructure investigations in the $5 s^{2} 5 p^{3}$ and $5 s^{2} 5 p^{2} 6 s$ configurations of ${ }^{121,123}$ Sb. Z Phys A 1978;288:247-56.

[5] Sobolewski LM, Bouazza S, Windholz L, Kwela J. Isotope shifts in Sb I. J Opt Soc Am B 2016;33:1921-7.

[6] Kramida A, Ralchenko Y, Reader J, NIST ASD Team. NIST atomic spectra database (version 5.4). Gaithersburg, MD: National Institute of Standards and Technology; 2016. Available: https://www.nist.gov/pml/ atomic-spectra-database.

[7] Cowan RD. The theory of atomic structure and spectra. University of California Press; 1981.

[8] Wilson M. Ab initio calculation of isotope shifts in ce II. Physica C 1978;95:129-33.

[9] Palmeri P, Quinet P, Bouazza S. MCDHF calculations of isotope shifts of evenparity fine-structure levels in neutral osmium. J Quant Spectrosc Radiat Transf 2016;185:70-8.

[10] Filippin L, Bieroń J, Gaigalas G, Godefroid M, Jönsson P. Multiconfiguration calculations of electronic isotope-shift factors in Zn I. Phys Rev A 2017;96:042502.

[11] Jönsson P, Gaigalas G, Bieroń J Froese Fischer C, Grant IP. New version: GRASP2k relativistic atomic structure package. Comput Phys Commun 2013; 184:2197-203.

[12] Nazé C, Gaidamauskas E, Gaigalas G, Godefroid M, Jönsson P. RIS3: A program for relativistic isotopic shift calculations. Comput Phys Commun 2013;183:2187-96.

[13] Shabaev MV. Mass correction in a strong nuclear field. Theor Math Phys 1985:63:588-96.

[14] Shabaev MV. QED theory of the nuclear recoil effect in atoms. Phys Rev A 1998;57:59-67.

[15] Palmer CWP. Reformulation of the theory of the mass shift. J Phys B 1987;20:5987-96.

[16] Grant IP. Methods of computational chemistry. New York: Plenum Press; 1988. $2,1$.

[17] Froese Fischer C, Godefroid M, Brage T, Jönsson P, Gaigalas G. Advanced multiconfiguration methods for complex atoms: I. Energies and wave functions. J Phys B: At Mol Opt Phys 2016;49:182004.

[18] Parpia FA, Mohanty AK. Relativistic basis-set calculations for atoms with fermi nuclei. Phys Rev A 1992:46:3735-45.

[19] Olsen J, Roos BO, Jørgensen P, Jensen HJA. Determinant based configuration interaction algorithms for complete and restricted configuration interaction spaces. J Chem Phys 1988;89:2185-92.

[20] Coursey JS, Schwab DJ, Tsai JJ, Dragoset RA. Atomic weights and isotopic compositions (version 3.0), Gaithersburg, MD: National Institute of Standards and Technology; 2015. [http://www.physics.nist.gov/pml/data/comp.cfm (last accessed December 18)].

[21] Li JG, Nazé C, Godefroid MR, Gaigalas G, Jönsson P. On the breakdown of the dirac kinetic energy operator for estimating normal mass shifts. Eur Phys J D 2012;66:290.

[22] Veitia A, Pachucki K. Nuclear recoil effects in antiprotonic and muonic atoms. Phys Rev A 2004;69:042501.

[23] Kozlov M.G., Korol V.A.. Relativistic and correlation corrections to the isotope shift in Ba II and Ba I. unpublished work; (http://www.qchem.pnpi.spb. ru/kozlovm/My_papers/notes/IS_in_Ba.pdf).

[24] Carette T, Drag C, Scharf O, Blondel C, Delsart C, Froese Fischer C, Godefroid MR. Isotope shift in the sulfur electron affinity: observation and theory. Phys Rev A 2010;81:042522.

[25] Hassini F, Ben Ahmed Z, Robaux O, Vergès J, J-F W. Study of fine and hyperfine structures in the spectrum of neutral antimony $\left({ }^{121} \mathrm{Sb}\right.$ I). J Opt Soc Am B 1988;5:2060.

[26] Filippin L, Beerwerth R, Ekman J, Fritzsche S, Godefroid M, Jönsson P. Multiconfiguration calculations of electronic isotope shift factors in al i. Phys Rev A 2016:94:062508.

[27] Filippin L, Godefroid M, Ekman J, Jönsson P. Core correlation effects in multiconfiguration calculations of isotope shifts in Mg I. Phys Rev A 2016;93:062512.

[28] Verdebout S, Rynkun P, Jönsson P, Gaigalas G, Froese Fischer C, Godefroid M. A partitioned correlation function interaction approach for describing electron correlation in atoms. J Phys B: At Mol Opt Phys 2013;46:085003. 